\title{
HIGH-RESOLUTION FIBER-COUPLED INTERFEROMETRIC POINT SENSOR FOR MICRO- AND NANO-METROLOGY
}

\author{
Markus Schake, Markus Schulz and Peter Lehmann \\ Dept. of Electrical Engineering, Chair in Measurement Technology, University of Kassel, \\ Wilhelmshoeher Allee 71, 34109 Kassel, Germany
}

\begin{abstract}
The determination of surface roughness is a common challenge in industrial quality assurance. Because tactile techniques like the stylus method or atomic force microscopy run the risk of damaging the measurement object there is a high demand for contact free optical measurements. In this contribution we demonstrate the feasibility of a high resolution fibercoupled interferometric point sensor with periodical path length modulation to determine the surface profile of rough surfaces. Measurements on two specimens characterized by different roughness parameters are presented and corrections for common measurement errors, due to phase ambiguity are discussed.
\end{abstract}

Index Terms - Fiber-sensor, Interferometry, Micro optical probe head, Roughness measurement, Path length modulation, Dual wavelength

\section{INTRODUCTION}

Modern industrial processes involving the fabrication of products which feature complex surface geometry and small tolerances, like optical lenses for use in cameras or smart phones and also injection valves for efficient combustion engines, require precise, process integrated measurement technology. Tactile sensors which are commonly used in industrial fabrication processes suffer from a limited scan rate due to the mechanical interaction with the measurement object and may cause damage to the investigated surface. Therefore, there is a high demand for contact-free, high-resolution, high-speed measurement systems which also satisfy the requirements to robustness and flexibility related to industrial inline applications. There is steady progress in the development of interferometric systems for surface measurements. However, chromatic confocal techniques are commonly used in this context too [2]. They are based on the effect of axial chromatic aberration, that results from the wavelength dependent refraction of light in a medium. A spectrally broadband light beam is focused by a lens on the surface under investigation. Due to the aberration of the focal lens the different wavelength components are separated along the optical axis, so that only one wavelength is focused at a time. By spectral analysis of the reflected beam the distance between sensor head and measurement object can be obtained [2]. An interferometric aproach based on a fiber optical Fizeau interferometer with an adapted Michelson interferometer was introduced in [1]. In this setup the fiber-optical Fizeau interferometer serves as a miniaturized fiber coupled sensing probe, applicable for use in an industrial environment. The adapted conventional Michelson interferometer is used to obtain low-coherence interferograms. These are generated by tilting one of the mirrors in a Michelson interferometer and recorded by a CCD line. Thus, the setup does not employ any mechanical movement [1]. A further well known and frequently used method for optical surface measurement is based on the principle of Phase Shifting Interferometry (PSI). 
PSI is a method to reconstruct the phase angle $\theta$ of an interference signal by sampling multiple images of the interference pattern which differ from each other by a known phase shift $\Delta \varphi$. Commonly, a linear change of phase in steps of $\Delta \varphi$ is applied by displacement or tilting of a reference mirror during data acquisition. For each phase shift value an intensity value of CCD image is recorded and stored. The initial phase $\theta$ depends on the surface height of the object under investigation and is estimated by a least squares method exploiting the sampled interference intensity $[3,9]$.

In this contribution we demonstrate the feasibility of a fiber-coupled interferometric point sensor with sinusoidal path length modulation and phase retrieval to measure rough surfaces. The working principle and the general capability of this sensor configuration was already published $[13,7]$. However, the measuring objects investigated so far were characterized by specularly reflecting surfaces of well known deterministic geometry such as sinusoidal surfaces, chirp standards, and rectangular grooves of well-known groove depth. A general difficulty of interferometric measurement of rough surfaces is the speckle effect, which leads to phase ambiguity. As a consequence, interferometric sensors which evaluate the phase of an occurring interference signal often fail on rough surfaces as it was reported previously by Vorburger et al. [14]. Therefore, this contribution is intended to study the applicability of our interferometric point sensor to surface roughness measurement.

\section{FIBER-COUPLED INTERFEROMETRIC POINT SENSOR}

The measurement technology group at the University of Kassel (Faculty of Electrical Engineering and Computer Science) deals with interferometric sensors for surface micro- and nanostructure and for form measurement [7]. One of the sensors developed over the last years is a fiber-coupled interferometric point sensor for micro- and nano-metrology that meets basic industrial requirements because of its miniaturized design and its robust measurement algorithm $[13,7]$. A schematic diagram of the setup and a picture of the optical probe head are shown in figure (1).

As displayed in the schematic diagram of figure (1) a) the experimental setup consists of two laser diodes $\mathrm{LD}_{1}$ and $\mathrm{LD}_{2}$ and photo diodes $\mathrm{PD}_{1}$ and $\mathrm{PD}_{2}$ which are in pairs connected to an optical Y-Coupler followed by a Wavelength Division Multiplexer (WDM) and the probe head. Monochromatic light from the laser diodes emitting at wavelengths in the infrared spectrum is coupled into a single mode fiber, passes the Y-Coupler and gets combined in the WDM. From there the light is guided to the micro-optical probe head.

As shown in figure (1) b) the optical probe consists of a glass ferrule by which the end of the fiber is adjusted with respect to a graded index lens which focuses the light on the measurement object. The optical probe is mounted to a piezo actuated bending beam. Expansion and contraction of the piezo crystal cause the probe head to oscillate perpendicularly to the measurement plane and therefore a periodical path length modulation occurs. Part of the incoming light is reflected inside the probe head and directed back to the fiber acting as the reference wave of the common path interferometer. The remaining light hits the surface of the measurement object and gets reflected there. Inside the fiber the light reflected by the measurement object and the light of the reference beam interfere. The interfering light is split up in its separate wavelength components by the WDM and interferograms are recorded as electrical signals by the photo diodes. Due to the periodic path length modulation the interference signals show a periodic intensity modulation too. 


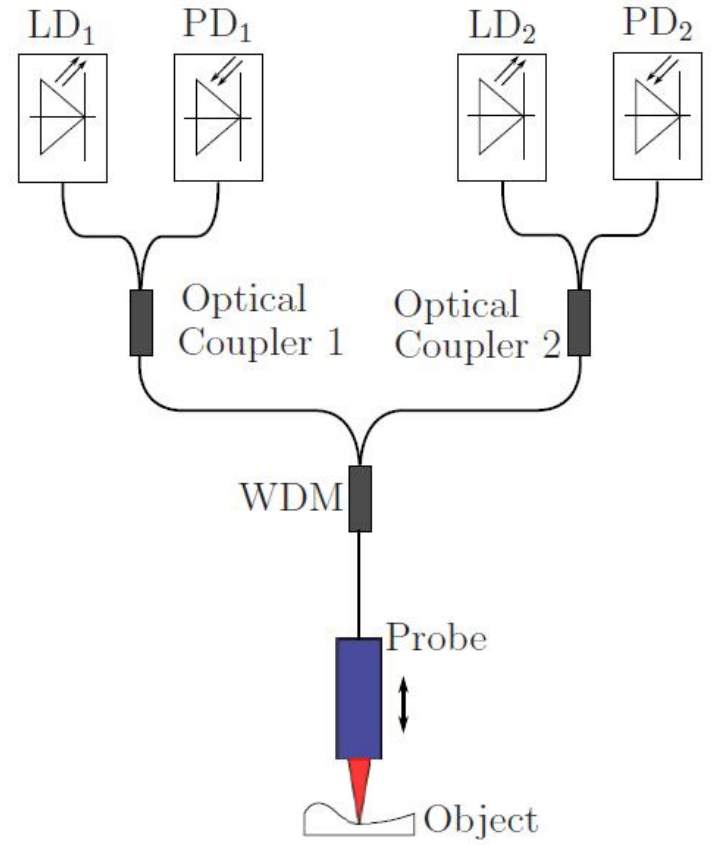

(a)

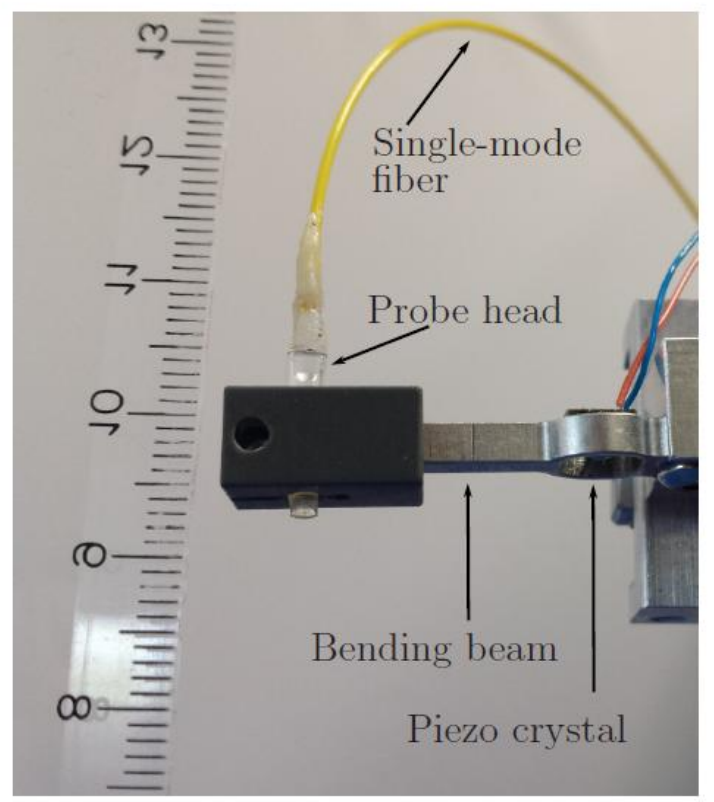

(b)

Figure (1): a) Schematic diagram of the dual wavelength common path interferometer setup, b) close-up view of the probe head with piezo actuator and bending beam.

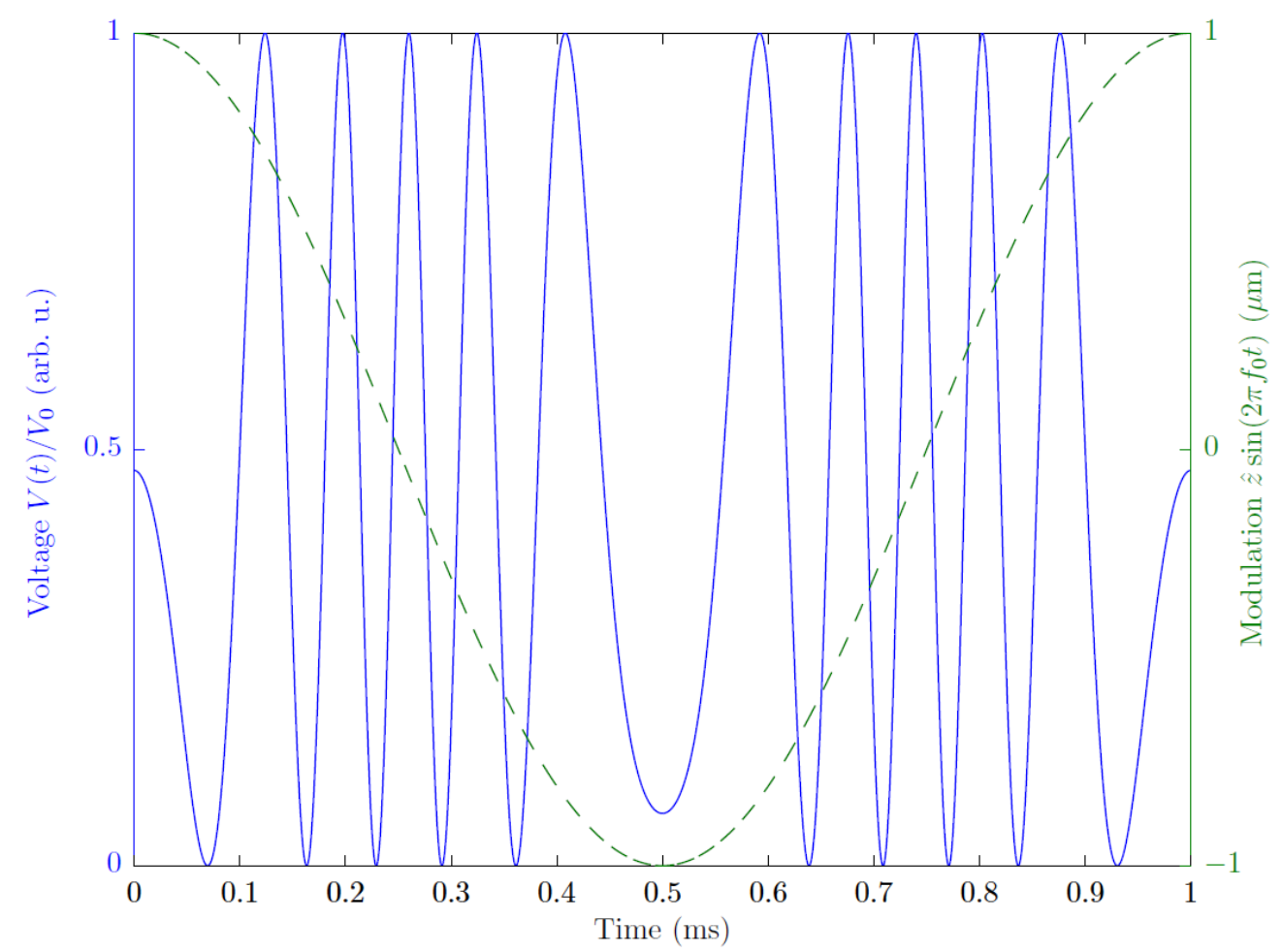

Figure (2): Simulated interference signal assuming $=1550 \mathrm{~nm}$ (solid line), actor movement causing a path length modulation of $f_{0}=1000 \mathrm{~Hz}$ and $\hat{z}=1 \mu \mathrm{m}$ [7] (dashed line). 
With a sinusoidal actor oscillation the interferogram received at the photodiodes can be described as

$$
V(t)=V_{0} \cos \left(\frac{4 \pi}{\lambda}\left\{\hat{z} \sin \left(2 \pi f_{0} t\right)+z(t)\right\}\right) .
$$

There $V_{0}$ represents the amplitude of the photo diode voltage, $\hat{z}$ is the maximum actor displacement, $f_{0}$ the actor frequency and $z(t)$ is the height of the surface under investigation which may change during the time [13].

Figure (2) shows a simulated interference signal recorded by the photodiode assuming a constant $z(t)$. For the simulation a wavelength of $\lambda=1550 \mathrm{~nm}$, a modulation frequency $f_{0}=1000 \mathrm{~Hz}$ and an actor displacement of $\hat{z}=1 \mu \mathrm{m}$ have been presumed. It can be observed, that there is a discontinuity of the phase vector at the actors turning points. Therefore, only sample points from the area of almost linear actor movement on the flanks of the actor signal are used for phase analysis.

A distance change between the probe head and the measurement object can be measured as a phase shift of the interference signal in each half cycle of the actor signal. The phase information is obtained by using a discrete Fourier transform algorithm at a single frequency.

\section{DETERMINATION OF SURFACE ROUGHNESS}

A common challenge in metrology is the determination of surface roughness. In ref. [14] lineprofiling tactile stylus methods are compared with different optical areal methods. Phaseshifting interferometry, white-light interferometry and confocal microscopes were used to determine the roughness average value $R_{a}$ of multiple frequently used roughness standards. In this contribution the feasibility of our optical, line-profiling, fiber-coupled, interferometric point sensor to measure rough surface textures will be demonstrated. To ensure comparability of our results we abide by the international standards for roughness measurements described in ISO 4287 [5] and ISO 11562 [6]. To separate the roughness from the surfaces waviness a cut-off-filter described in [6] is used.The cut-off-filter's transfer function is given by:

$$
H(\omega)=\exp \left(-\pi\left(\frac{\alpha \lambda_{\mathrm{co}} \omega}{2 \pi}\right)^{2}\right) \text {. }
$$

Where $\omega$ represents the spatial frequency, $\lambda_{\text {co }}$ is the specimen specific cut-off-wavelength that acts as a threshold value to distinguish between roughness and waviness and $\alpha=\sqrt{\frac{\log 2}{\pi}}$.

By multiplying the cut-off-filter according to equation (3.1) with the Fourier transformed height values and applying the inverse Fourier transform to the result the measurement object's waviness can be separated. By subtracting the waviness profile from the sampled height values the roughness profile will result. The standards $[5,6]$ also propose a traversing length $l_{t} \geq 6 \lambda_{c o}$ for which the sensor acquires samples and an evaluation length of $l=5 \lambda_{c o}$ where the evaluation takes place after filters were applied.

In our experiment we focus on two commonly used parameters to describe surface roughness, the roughness average value $R_{a}$ also referred in [14] and the average maximum height of the profile $R_{z}$. The roughness average $R_{a}$ is defined as [5,8]:

$$
R_{a}=\frac{1}{N} \sum_{k=1}^{N}|z(k)| \text {. }
$$

The required amount of samples $N$ can be calculated by the evaluation length $l$, the velocity $v$ of the scanning axis, and the data rate $f_{d}$ of the applied sensor.

$$
N=\operatorname{round}\left(\frac{l \cdot f_{d}}{v}\right)
$$

The average maximum height $R_{z}$ is defined as [5]: 


$$
R_{z}=\frac{1}{n} \sum_{i=1}^{n} R_{z i}
$$

To calculate $R_{z}$ the evaluation length is departed into $n$ equal sections. The value $R_{z i}$ is given as the maximum roughness depth

of the $i_{t h}$ measurement section.

$$
R_{z i}=\left(\max _{z} z(k)-\min _{z} z(k)\right) \forall k \in\left[(i-1) \frac{N}{n}+1, i \frac{N}{n}\right]
$$

\section{RESULTS OF OPTICAL AND TACTILE MEASUREMENT}

The fiber-coupled interferometric point sensor shown in figure (1) b) was used to investigate the surface roughness of two roughness standards with different roughness averages $R_{a}$ and average maximum heights $R_{z}$. The first specimen is the PTB roughness standard 626 . The standard 626 is characterized by the roughness average value $R_{a}=483 \mathrm{~nm}$ and the average maximum height $R_{z}=3290 \mathrm{~nm}$ [11]. The PTB measurements were carried out using a tactile probe with a stylus tip radius of $5 \mu \mathrm{m}$ and applying a profile filter with a wavelength limit of $\lambda_{\text {co }}=0.8 \mathrm{~mm}$. The used evaluation length was $5 \lambda_{\text {co }}=4 \mathrm{~mm}$ and the sampling interval was $0.2 \mu \mathrm{m}$. In addition to the calibration certificate [11] the PTB also supplied us with a profile record of the standard 626. The roughness profile is depicted in figure (3) a).

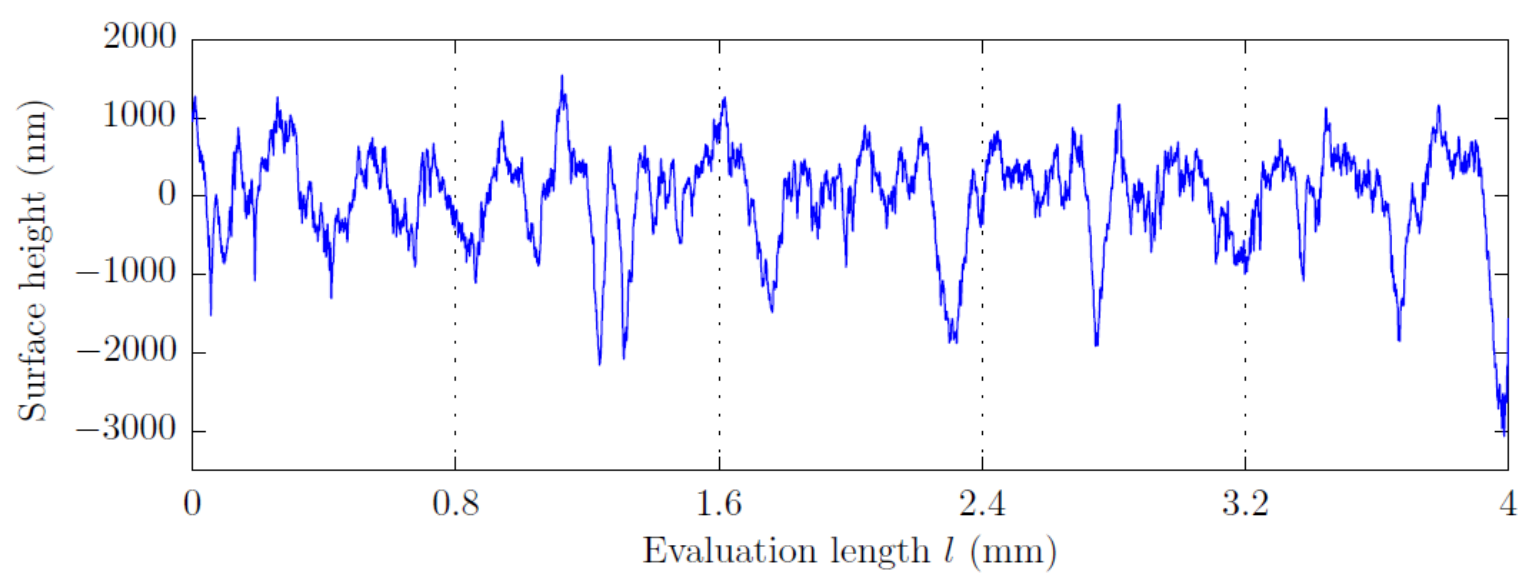

a)

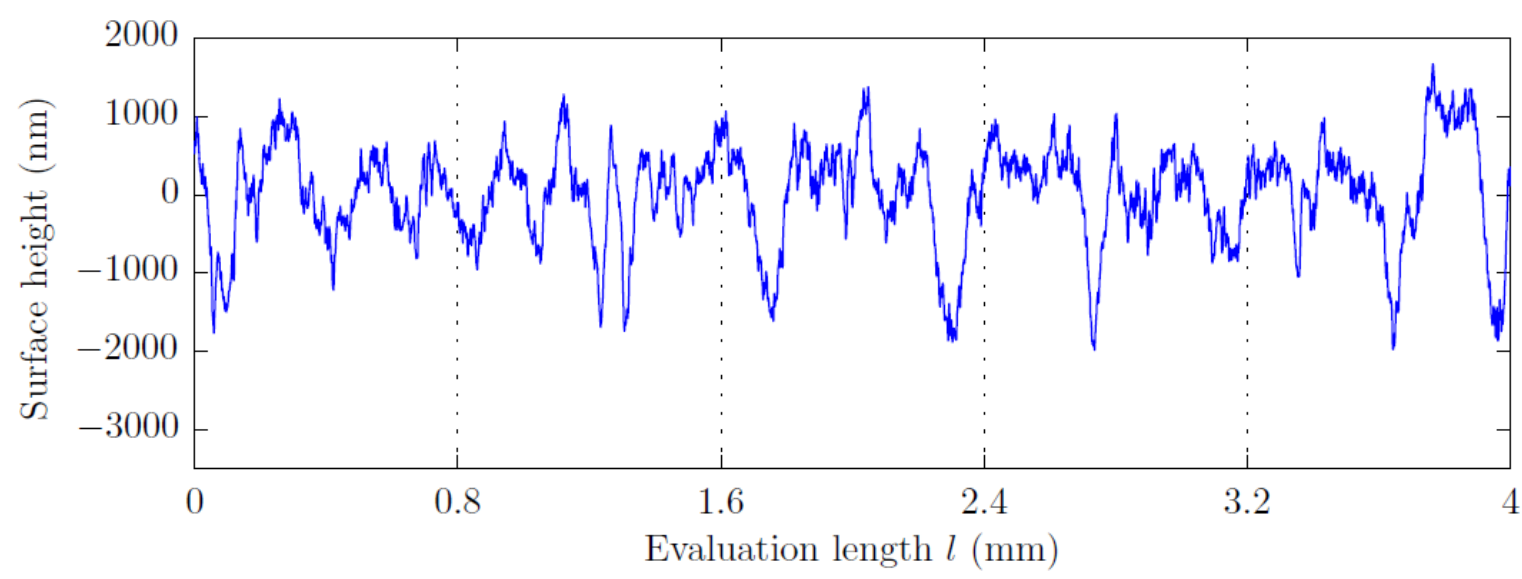

b)

Figure (3): a) Standard No. 626, calibration data obtained by stylus measurement conducted by PTB [11], b) Standard No. 626, surface profile obtained with the interferometric system described above [12].

Figure (3) b) shows the surface profile of the standard 626 measured with our interferometric point sensor. According to $[5,6]$ the same profile filter $\lambda_{\text {co }}$ and evaluation length was used as 
in the PTB setup. The scanning velocity was $v=0.05 \mathrm{~mm} / \mathrm{s}$ while taking samples with a frequency of $f_{d}=1080 \mathrm{~Hz}$ resulting in a sampling interval of approximately $0.05 \mu \mathrm{m}$. The laser wavelength $\lambda_{1}=1310 \mathrm{~nm}$ and a probe head with a numerical aperture of $N A=0.4$ were used. The roughness parameters determined by equations (3.2) and (3.4) are $R_{a}=503$ $\mathrm{nm}$ and $R_{z}=3185 \mathrm{~nm}$.

The second specimen is the standard No. 6633 produced by the company Halle PräzisionsKalibriernormale $\mathrm{GmbH}$ corresponding to PTB-standards. The standard 6633 is characterized by the roughness average value $R_{a}=1650 \mathrm{~nm}$ and the average maximum height

$R_{z}=7960 \mathrm{~nm}$ [4]. The in-house calibration measurements of Halle were carried out using a tactile probe with a stylus tip radius of $2 \mu \mathrm{m}$ and applying a profile filter with a wavelength limit of $\lambda_{\text {co }}=0.8 \mathrm{~mm}$. The used evaluation length was $5 \lambda_{\text {co }}=4 \mathrm{~mm}$ and the sampling interval was $0.5 \mu \mathrm{m}$. In addition to the calibration certificate [4] Halle also supplied us with a profile record of the standard 6633. The roughness profile is depicted in figure (4) a).

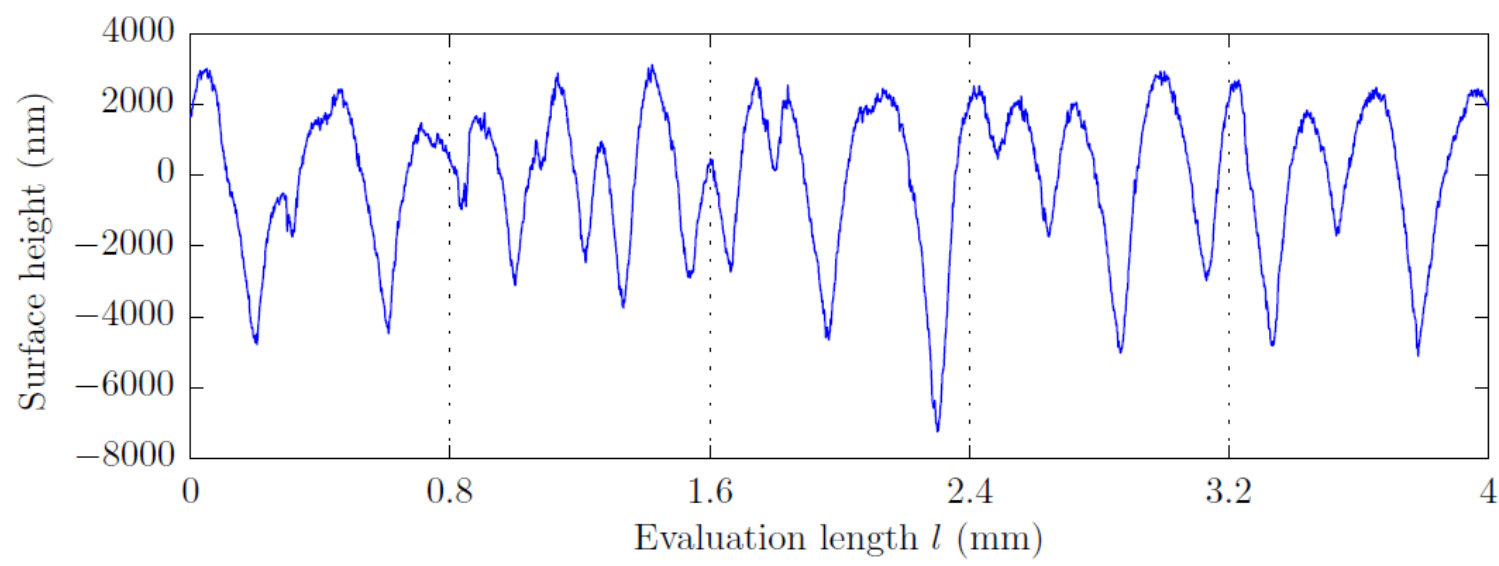

a)

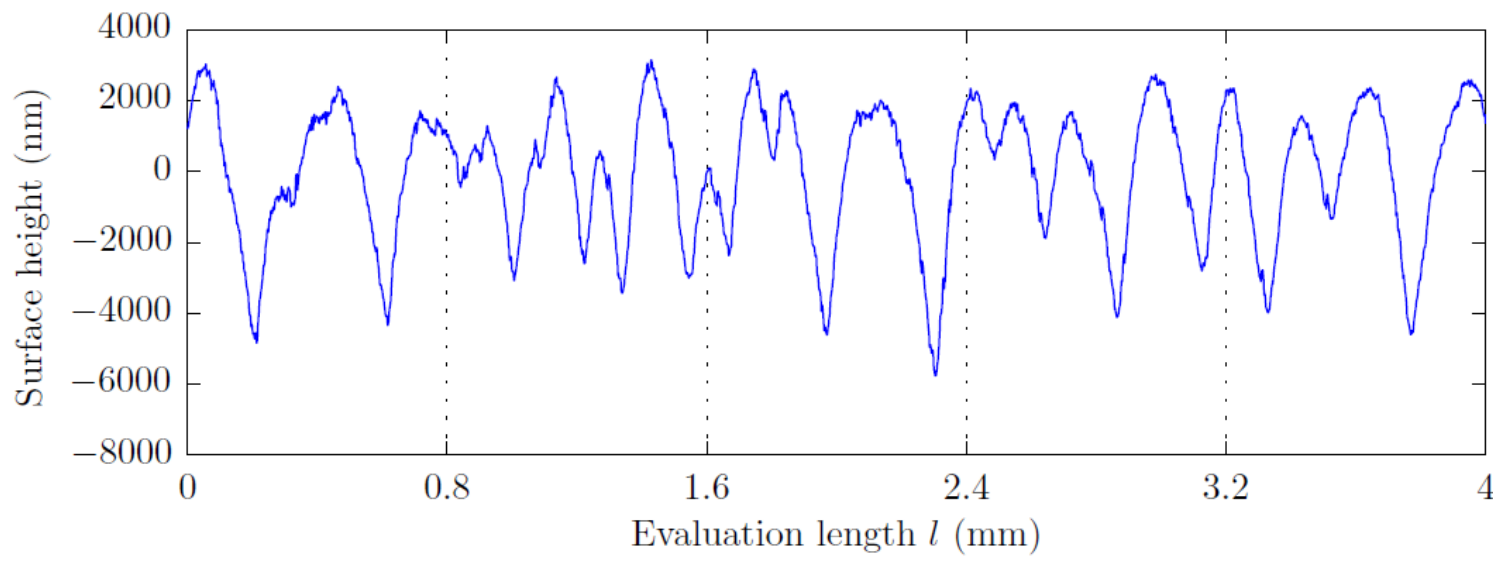

b)

Figure (4): a) Standard No. 6633, calibration data obtained by stylus measurement conducted by Halle [4], b) Standard No. 6633, surface profile obtained with the interferometric system described above.

Figure (4): b) shows the surface profile of the standard 6633 measured with our interferometric point sensor. According to $[5,6]$ the same profile filter $\lambda_{\text {co }}$ and evaluation length was used as in the PTB and Halle setup. The scanning velocity was $v=0.4 \mathrm{~mm} / \mathrm{s}$ while taking samples with a frequency of $f_{d}=1000 \mathrm{~Hz}$ resulting in a sampling interval of approximately $0.4 \mu \mathrm{m}$. The used laser wavelength was $\lambda_{2}=1550 \mathrm{~nm}$. The roughness parameters determined by equations (3.2) and (3.4) are: $R_{a}=1568 \mathrm{~nm}$ and $R_{z}=7429 \mathrm{~nm}$. 
Table (1) gives a summary of the measured roughness parameters and the relative deviation between optical and tactile methods.

\begin{tabular}{|l|c|c|}
\hline Standard type & (PTB) 626 & (Halle) 6633 \\
\hline$R_{\mathrm{a}, \text { tactile }}(\mathrm{nm})$ & 483 & 1650 \\
\hline$R_{\mathrm{z}, \text { tactile }}(\mathrm{nm})$ & 3290 & 7960 \\
\hline$R_{\mathrm{a}, \mathrm{optic}}(\mathrm{nm})$ & 503 & 1568 \\
\hline$R_{\mathrm{z}, \mathrm{optic}}(\mathrm{nm})$ & 3185 & 7429 \\
\hline$\frac{R_{\mathrm{a}, \mathrm{optic}}-R_{\mathrm{a}, \text { tactile }}}{R_{\mathrm{a}, \text { tactile }}}$ & $4.1 \%$ & $-5.0 \%$ \\
\hline$\frac{R_{\mathrm{z}, \mathrm{optic}}-R_{\mathrm{z}, \text { tactile }}}{R_{\mathrm{z}, \text { tactile }}}$ & $-3.2 \%$ & $-6.7 \%$ \\
\hline
\end{tabular}

Table (1): Summary of surface roughness parameters for tactile stylus and optical interferometric measurements.

\section{DISCUSSION OF THE RESULTS}

As it can be obtained from figure (3) and (4) the measurement of the roughness standards with $R_{a}=483 \mathrm{~nm}$ and $R_{a}=1650 \mathrm{~nm}$, conducted by tactile and optical methods are in good agreement. All details of the surface profiles can be found in both, the tactile and the interferometric measurement results. The maximum relative deviation in the $R_{a}$ values determined by stylus and interferometric measurement is about $5 \%$, which is typically good enough for surface roughness measurement.

Although there is a good congruence in general, still discrepancies occur, especially at high slopes and deep, narrow valleys in the surface structure. At high surface slopes between two sampling points, a height difference of more than $\pm \lambda_{i} / 4$ may occur that would cause ambiguity in the phase-unwrapping and therefore lead to height measurement errors.

Ref. [10] introduces a technique to correct for phase jumps, referred to as "ghost steps," by using the surface height information generated from the interferogram of a second light source emitting at a different wavelength. If ambiguity issues occur for the wavelength $\lambda_{1}, \lambda_{2}$ or both this will result in a ghost step with step height of an integer multiple of $\frac{\lambda_{1}}{2}, \frac{\lambda_{2}}{2}$ or $\frac{\lambda_{2}-\lambda_{1}}{2}$ [10].

As described in section (2) our fiber-coupled interferometric point sensor features two light sources of $\lambda_{1}=1310 \mathrm{~nm}$ and $\lambda_{2}=1550 \mathrm{~nm}$ and is capable of conducting height measurements with both wavelengths simultaneously. If the surface profile is measured correctly by evaluating both wavelengths the difference between the two height profiles is approximately zero. If ghost steps occur for one or both wavelengths this results in a step in the difference of the profiles. The step height can be interpreted as described above. 


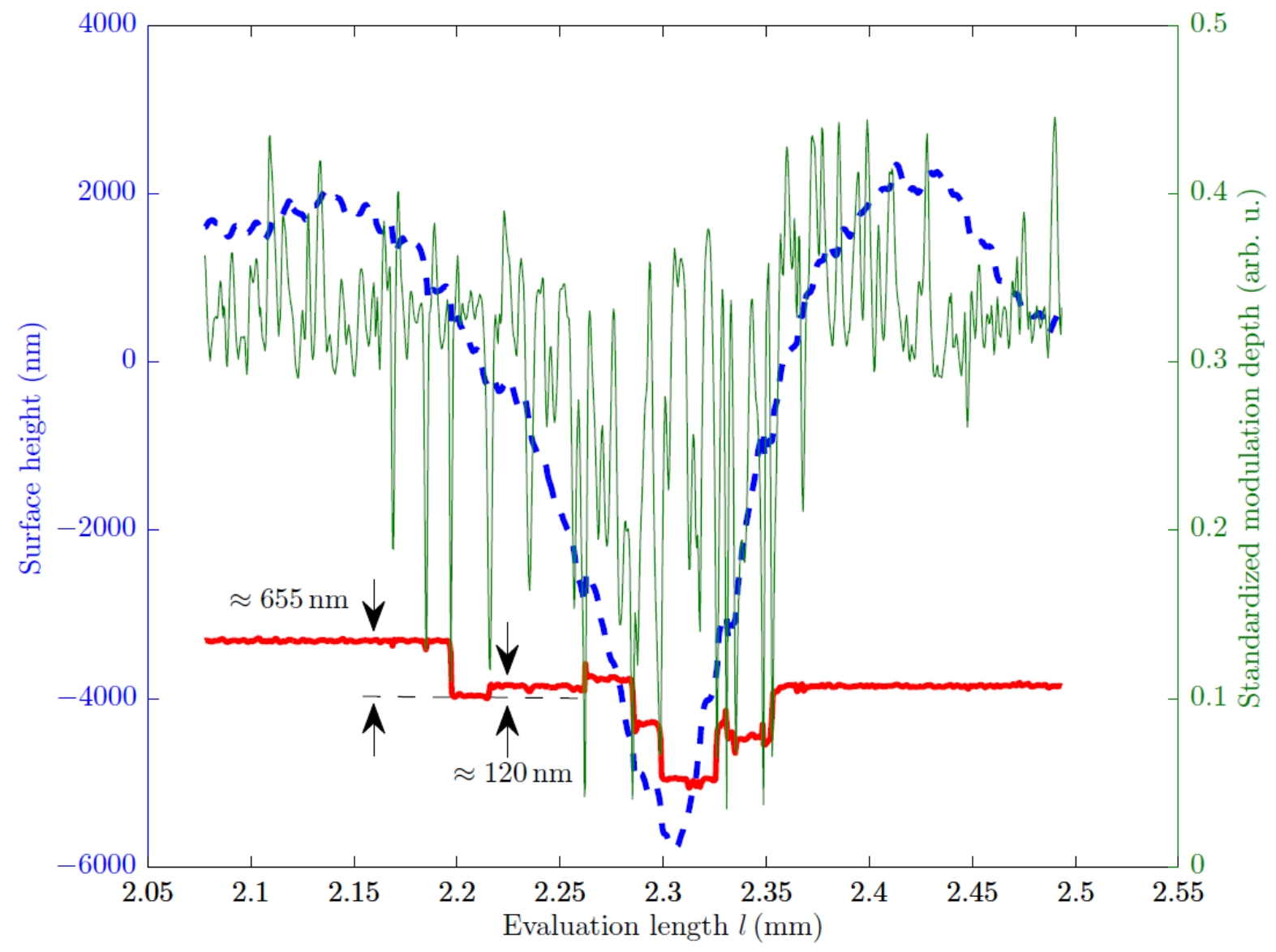

Figure (5): Zoomed interval of the surface profile of standard No. 6633 depicted in figure (4) b) in the interval of $[2.1,2.5] \mathrm{mm}$ (dashed line), difference of the unfiltered surface height values measured by the interferometric point sensor employing the two different wavelengths $\lambda_{1}=1310 \mathrm{~nm}$ and $\lambda_{2}=1550 \mathrm{~nm}$ (bold, solid line), corresponding modulation depth of the sampled interference signal of wavelength $\lambda_{2}$ from which the height values were calculated (slim, solid line).

The dashed line in figure (5) shows a detailed view of the profile of the roughness standard 6633 according to figure (4) b) on the interval of $[2.1,2.5] \mathrm{mm}$. As it can be seen in figure (4) this interval contains the deepest valley along the evaluation length and shows high slopes. The bold solid line in figure (5) indicates the difference of the unfiltered surface height profiles corresponding to the wavelengths $\lambda_{1}=1310 \mathrm{~nm}$ and $\lambda_{2}=1550 \mathrm{~nm}$ on the same interval. It can be observed that multiple ghost steps occur whose height correspond to integer multiples of $\frac{\lambda_{1}}{2}=655 \mathrm{~nm}, \frac{\lambda_{2}}{2}=775 \mathrm{~nm}$ and $\frac{\lambda_{2}-\lambda_{1}}{2}=120 \mathrm{~nm}$. Two of the ghost steps are indicated by arrows in figure (5).

Furthermore, this result demonstrates that a robust phase evaluation is possible for nearly all measured signals. This is an important prerequisite for the application of algorithms that identify and reduce phase steps resulting from $2 \pi$ ambiguity problems. The slim, solid line in figure (5) shows the standardized modulation depth of the sampled interference signal of wavelength $\lambda_{2}$, from which the height values were calculated on the corresponding interval. The reference value for standardized modulation depth was found empirically as the maximum peak to peak amplitude of the interferogram of a plane mirror.

It can be seen, that the modulation depth of the interference signal is less than half of the reference value of a plane and smooth highly reflective surface. This is due to the diffuse 
scattering of the light at the rough surface. As shown in figure (5) the modulation depth rapidly decreases when the probe passes areas of highly sloped surface structures. Also the occurrence of ghost steps seems to corresponds to low modulation depths of the related signals. The decreased modulation depth and the algorithm proposed in [10] may be used to correct the surface profile by first identifying the ghost steps and then assigning them with respect to their step height to one of the three cases of ambiguity issues described above. Due to the rather high numerical aperture of our probe head of $N A=0.4$, that generally enables the sensor to measure even sloped surfaces, the light is strongly focused. To obtain a high light intensity at the photodiodes and a high modulation depth, the light should be focused on the surface of the measurement object. In the case of rough surfaces with high roughness value $R_{a}$, like the standard 6633, parts of the surface profile may be out of focus due to the height changes. At extreme points like high peaks and deep valleys on the standard the focus point lies above or beyond the measurement object's surface and therefore the measurement spot is widened and increasing in diameter. This causes a decrease of the lateral resolution and a loss of intensity due to scattering at the rough surface.

Therefore it is mandatory to search for a good focal point on the surface in order to obtain reliable roughness measurement results. Consequently, a further improvement of the experimental setup would be given by a linear stage maintaining the position of the probe during measurement to keep the light focused on the surface of the measuring object.

\section{CONCLUSION}

It has been demonstrated that the fiber-coupled, interferometric point sensor for micro- and nano-metrology presented in this contribution is feasible to measure rough surfaces of $R_{a}=483 \mathrm{~nm}$ and $R_{a}=1650 \mathrm{~nm}$. In contrast to the study of Vorburger et al. [14] our results indicate that robust measurement of surface roughness is still possible even with an interferometric sensor which is based on a phase evaluation technique. The major difference of our sensor principle compared to areal measuring PSI interferometers employed in ref. [14] is the point-wise measurement in combination with the fiber-coupled sensor head. Since the fiber end face is imaged on the surface under investigation, the sensor acts as a confocal sensor in the sense that only light from a very limited spatial region contributes to the signal formation. Compared to the referred tactile stylus method the interferometric sensor encounters some difficulties by measurement of highly sloped surfaces where phase ambiguities can occur. A possible countermeasure presented in [10] could improve the behavior of the sensor. Furthermore, the high numerical aperture of the probe, necessary to measure high slopes, causes a strong focus of the light and therefore shows a limited depth of focus. Hence, a tracking system is required to keep the probe head in an optimal distance to the measurement object and to prevent the loss of intensity. The contact free measurement and the high data rate of the device enable fast surface measurements with high spatial resolution. Further information concerning the interferometric point sensor and its feasibility for different applications can be found in $[13,7]$. 


\section{REFERENCES}

[1] F. Depiereux, P. Lehmann, T. Pfeifer und R. Schmitt, " Fiber-optical sensor with miniaturized probe head and nanometer accuracy based on spatially modulated low- coherence interferogram analysis," Applied Optics, Vol. 46, No. 17, pp. 3425-3431, 2007

[2] C. Dietz, M. Jurca, "Eine Alternative zum Laser, Ein Weißlicht-Messverfahren dringt in den Sub- $\mu$ m-Bereich ein," Sensor Magazine Nr. 4, pp. 13-15, 1997

[3] P. de Groot in R. Leach (Ed), "Optical Measurement of Surface Topography," Springer-Verlag Berlin Heidelberg, pp. 167-186, 2011

[4] Halle Präzisions-Kalibriernormale $\mathrm{GmbH}$, "In-house calibration certificate for roughness standard corresponding to PTB-Standard, Line of products: KNT 4058/03, Serial No.: 6633," 2014

[5] International Organization for Standardization, "ISO 4287 (1997) Geometrical product specifications (GPS) - Surface texture: profile method-terms, definitions and surface texture parameters,"

[6] International Organization for Standardisation, "ISO 11562 (1997) Geometrical Product Specifications (GPS) - Surface texture: Profile method - Metrological characteristics of phase correct filters,"

[7] H. Knell, M. Schake, M. Schulz and P. Lehmann, "Interferometric sensors based on sinusoidal optical path length modulation," SPIE Proceedings Vol. 9132: Optical Micro- and Nanometrology V, 2014

[8] K. Leonhardt, K. H. Rippert und H. J. Tiziani, "Optische Mikroprofilometrie und Rauheitsmessung," Technisches Messen, 54. Jahrgang, Heft 6, pp. 243-252, 1987

[9] Cruz Meneses-Fabian and Uriel Rivera-Ortega, "Phase-Shifting Interferometry by Amplitude Modulation," InTech, Published online, pp. 171-195, 2012

[10] Jan Niehues, Peter Lehmann and Klaus Bobey, "Dual-wavelength vertical scanning low-coherence interferometric microscope," Applied Optics, Vol. 46, No. 29, pp. 7141-7148, 2007

[11] Physikalisch-Technische Bundesanstalt, "Calibration Certificate for Roughness measurement standard type 626," 2009

[12] Markus Schulz, "Interferometrisch messender faseroptischer Sensor mit mechanisch oszillierender Sonde, " Dissertationsschrift in Vorbereitung, Universität Kassel, FB16, Messtechnik, 2014

[13] M. Schulz and P. Lehmann, "Measurement of distance changes using a fibre-coupled common path interferometer with mechanical path length modulation," Measurement Science and Technology, Vol. 24 (2013) 065202 (8pp)

[14] T.V. Vorburger, H. G. Rhee, T. B. Renegar, J. F. Song, A. Zheng, "Comparison of optical and stylus methods for measurement of surface texture," Int. J. Adv. Manuf. Technol. Vol. 33, pp. 110-118, 2007

\section{CONTACT}

Dipl.-Ing. M. Schake

Dipl.-Ing. M. Schulz

Prof. Dr.-Ing. habil. P. Lehmann markus.schake@uni-kassel.de markus.schulz@uni-kassel.de p.lehmann@uni-kassel.de 